\title{
Quality Evaluation of Traditional Chinese Drug Toad Venom from Different Origins through a Simultaneous Determination of Bufogenins and Indole Alkaloids by HPLC
}

\author{
Ping Zhang, ${ }^{a}$ Zheng Cui, ${ }^{*}, a$ Yashu Liu, ${ }^{a}$ Dong Wang, ${ }^{a}$ Na Liv,,${ }^{a}$ and Masayuki YoshiKaWA ${ }^{b}$ \\ ${ }^{a}$ Pharmacognosy Department of Traditional Chinese Medicine College, Shenyang Pharmaceutical University; 103 \\ Wenhua Road, Shenyang, 110016, China: and ${ }^{b}$ Kyoto Pharmaceutical University; Misasagi, Yamashina-ku, Kyoto \\ 607-8412, Japan. Received June 8, 2005; accepted July 25, 2005
}

\begin{abstract}
A simple and efficient HPLC method has been developed to evaluate the quality of traditional Chinese medicine toad venoms. The major bioactive ingredients, including 10 bufogenins and 4 indole alkaloids in the drug, were separated and quantified on a phenyl-hexyl column with a UV detector. A total of 27 toad venom samples from two species, Bufo bufo gargarizans CANTOR and Bufo melanostictus SCHNEIDER, from the different drug production regions of China, were analyzed. The chromatograms showed significant differences with respect to the samples from different origins. These toad venom samples can be distinctly classified into 4 groups by cluster analysis using the contents of the 14 main constituents, including toad venom samples from $B$. bufo gargarizans from north China, median China and south China, and samples from B. melanostictus from south China. Toad venom samples from $B$. bufo gargarizans from median China were considered to be of the highest quality.
\end{abstract}

Key words quality evaluation; HPLC; toad venom; Bufo bufo gargarizans; Bufo melanostictus

Toad venom, in the form of dried toad secretions derived from either Bufo bufo gargarizans CANTOR or B. melanostictus SCHNEIDER, has been widely used as a cardiotonic, diuretic, anodyne and antineoplastic agent in Asia. ${ }^{1)}$ Chemical studies of toad venom showed that bufotoxins, bufogenins and indole alkaloids were the main and characteristic constituents, ${ }^{2,3)}$ and that bufotoxins existing in the fresh secretion were almost hydrolyzed to their corresponding bufogenins during the drying process. ${ }^{4)}$ Bufogenin, a kind of bufadienolide steroid, was the major pharmacologically active substance, and exhibited a range of bioactivities, including as a cardiotonic, and in boosting blood pressure, exciting respiration, as an antineoplastic, and and as a surface anesthetic. ${ }^{5-7)}$ The indole-alkylamines existing in the frogs were reported to have vasoconstrictor, convulsion, hallucinogen and cytotoxicity activities. ${ }^{89}$ ) The analysis of bufogenins was accomplished by using TLC, HPLC and GC. ${ }^{10^{-12}}$ It was found that the contents of the bufogenins in commercial toad venom varied widely. ${ }^{13}$ ) The presence of abundant indole-alkylamines in the drug was ignored in previous studies, which could affect the safety of the drug. ${ }^{14)}$ This oversight could seriously affect the quality control and clinical utilization of toad venom.

In this paper, a HPLC method for simultaneous determination of the major bioactive bufogenins and indole alkaloids in toad venom is elaborated. Based on the baseline chromatographic separation of most of the constituents in toad venom on a phenyl-hexyl column with gradient elution, 10 bufogenins and 4 indole alkaloids in 27 toad venom samples were identified and quantitated, and the chromatograms thus produced reflect the differences of toad venom samples from different toad species and drug production regions. The determination of indole alkaloids in toad venom, as well as the influence of the species, regions and collection seasons, on the quality of the drug was investigated for the first time.

\section{Experimental}

General Experimental Procedures Chromatographic system: the es- sential parts of a Shimadzu LC 2010A consisted of a high-speed autosampler, a column oven, a UV detector set at $296 \mathrm{~nm}$ and a LC workstation for data collection. The HPLC separation was performed by a linear gradient elution program on a phenomenex Luna phenyl-hexyl analytical column $(250 \times 4.6 \mathrm{~mm}, 5 \mu \mathrm{m})$, along with a phenomenex phenyl-packed guard column $(4 \times 3.0 \mathrm{~mm}, 5 \mu \mathrm{m})$. The gradient elution was effected with a mixture of two eluents: (A) phosphate buffer $\left(20 \mathrm{~mm} \mathrm{NaH}_{2} \mathrm{PO}_{4}, 5 \mathrm{~mm}\right.$ citric acid, $0.2 \mathrm{~mm}$ heptanesulfonate, $\mathrm{pH} 4.0$ ) and (B) methanol; $0-30 \mathrm{~min}, 12 \% \mathrm{~B} ; 30$ $40 \mathrm{~min}, 12-50 \% \mathrm{~B} ; 40-90 \mathrm{~min}, 50-60 \% \mathrm{~B} ; 90-90.1 \mathrm{~min}, 60-12 \% \mathrm{~B}$. Equilibration time between each chromatographic run was $20 \mathrm{~min}$. The flow rate of the mobile phase was $1.0 \mathrm{ml} / \mathrm{min}$. The experiments were conducted at $40^{\circ} \mathrm{C}$.

Isolation of Standard Compounds The chloroform extraction $(40.0 \mathrm{~g})$ of toad venom (B. bufo gargarizans, $200.0 \mathrm{~g}$ ) was subjected to column chromatography on silica gel with a $\mathrm{CHCl}_{3}$-acetone gradient elution $(100: 0-$ $50: 50)$ to give fractions I-XII. Fractions II ( $3.5 \mathrm{~g})$ and III $(5.5 \mathrm{~g})$ were separated by semipreparative HPLC on an ODS column eluted with $\mathrm{MeOH}-$ $\mathrm{H}_{2} \mathrm{O}(7: 3)$, and were further purified by recrystallization to afford compounds $11(1.16 \mathrm{~g}), \mathbf{1 2}(1.35 \mathrm{~g})$ and $\mathbf{1 3}(1.24 \mathrm{~g})$. From fractions IV $(0.68 \mathrm{~g})$ and $\mathrm{V}(0.92 \mathrm{~g})$, compounds $7(0.15 \mathrm{~g}), \mathbf{8}(0.26 \mathrm{~g}), \mathbf{9}(0.12 \mathrm{~g})$ and $\mathbf{1 0}(0.11 \mathrm{~g})$ were obtained by a similar method with $\mathrm{MeOH}-\mathrm{H}_{2} \mathrm{O}(3: 2)$, and compounds $5(0.22 \mathrm{~g})$ and $7^{\prime}(0.05 \mathrm{~g})$ were gained from fractions VI $(0.94 \mathrm{~g})$ and VIII $(1.26 \mathrm{~g})$ with $\mathrm{MeOH}-\mathrm{H}_{2} \mathrm{O}(1: 1)$. The methanol extraction $(30.0 \mathrm{~g})$ of the residues was treated with a $\mathrm{HP}-20$ column by $\mathrm{MeOH}-\mathrm{H}_{2} \mathrm{O}(20: 80)$ to obtain the elution substance $(10.0 \mathrm{~g})$, and the elution substance was prepared by silica gel TLC chromatography to get fractions $1-5$ (butanol-ammonia- $\mathrm{H}_{2} \mathrm{O}$, $9: 1: 1)$. These fractions were further purified by the above ODS column $\left(\mathrm{CH}_{3} \mathrm{CN}-\mathrm{H}_{2} \mathrm{O}-\mathrm{TFA}, 10: 90: 0.05\right)$ to give compounds $\mathbf{1}(40 \mathrm{mg}), \mathbf{3}(20 \mathrm{mg})$, $4(60 \mathrm{mg}), \mathbf{4}^{\prime}(10 \mathrm{mg})$, and $\mathbf{4}^{\prime \prime}(24 \mathrm{mg})$. The methanol extraction $(1.0 \mathrm{~g})$ was directly subjected to preparative HPLC $\left(\mathrm{CH}_{3} \mathrm{CN}-\mathrm{H}_{2} \mathrm{O}-\mathrm{TFA}, 2: 98: 0.05\right)$ to afford compound $2(14 \mathrm{mg})$. The compound $14(30 \mathrm{mg})$ was isolated by the above ODS column ( $\left.\mathrm{MeOH}-\mathrm{H}_{2} \mathrm{O}, 5: 5\right)$ from the toad venom $(1.0 \mathrm{~g})$ of $B$. melanostictus. These separated compounds were purified to purity of greater than $98 \%$ and used as the standard compounds in our studies. The structures of these compounds were identified by determining ${ }^{1} \mathrm{H}-,{ }^{13} \mathrm{C}$-NMR and mass spectra, and by comparing their spectral data with the literature values. They are serotonin (1),${ }^{14)} N^{\prime}$-methyl serotonin $(\mathbf{2}),{ }^{14)}$ bufotenine $(\mathbf{3}),{ }^{9}$ bufotenidine (4), ${ }^{14)}$ bufotenine oxide $\left(4^{\prime}\right),{ }^{15)}$ bufobutanoic acid $\left(\mathbf{4}^{\prime \prime}\right),{ }^{9)}$ gamabufotalin $(5),{ }^{18)}$ arenobufagin $(6),{ }^{16)}$ hellebrigenin $(7),{ }^{17)}$ desacetylcinobufotalin $\left(7^{\prime}\right),{ }^{17)}$ bufotalin $(\mathbf{8}),{ }^{17)}$ telocinobufagin $(\mathbf{9}),{ }^{18)}$ cinobufotalin $(\mathbf{1 0}),{ }^{18)}$ bufalin $(\mathbf{1 1}),{ }^{17)}$ resibufogenin (12), ${ }^{17)}$ cinobufagin $(\mathbf{1 3})^{17)}$ and 19-hydroxy bufalin (14) ${ }^{17)}$ (Fig. 1).

Crude Drugs Ten batches of commercial toad venom (Linyi, Shandong) were provided and identified as the secretion of B. bufo gargarizans by the Shandong Institute for Drug Control, China. Seventeen toad venom samples were collected from living toads which were captured from different 
<smiles>[R]NCCc1c[nH]c2ccc(O)cc12</smiles><smiles>C[N+](C)(C)CCc1c[nH]c2ccc([O-])cc12</smiles>
$\begin{array}{lll} & \mathrm{R}_{1} & \mathrm{R}_{2} \\ 1 & \mathrm{H} & \mathrm{H} \\ 2 & \mathrm{H} & \mathrm{CH}_{3} \\ 3 & \mathrm{CH}_{3} & \mathrm{CH}_{3} \\ 4 " & \mathrm{COCH}_{2} \mathrm{CH}_{2} \mathrm{COOH} & \mathrm{H}\end{array}$

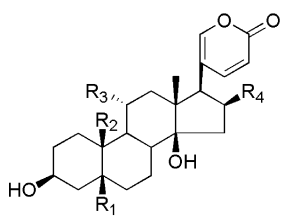<smiles>O=C1CC2(c3ccc(=O)oc3)CCCC2(O)C2CCC3C[C@@H](O)CC[C@]32C1</smiles><smiles>C[N+](C)(O)CCc1c[nH]c2ccc(O)cc12</smiles>

$4^{\prime}$

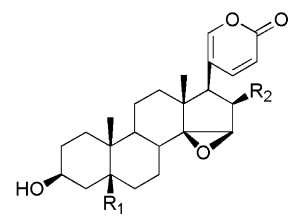

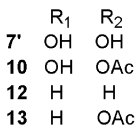

Fig. 1. Structures of Standard Compounds Isolated from Toad Venom

Table 1. Toad Venom Samples from Different Origins

\begin{tabular}{rlll}
\hline \hline $\begin{array}{c}\text { Sample } \\
\text { No. }\end{array}$ & \multicolumn{1}{c}{ Location } & Date & Species of the toads \\
\hline 1 & Qiqihaer, Heilongjiang & May, 2004 & B. bufo gargarizans \\
2 & Shenyang, Liaoning & May, 2004 & B. bufo gargarizans \\
3 & Shenyang, Liaoning & Aug., 2004 & B. bufo gargarizans \\
4 & Shenyang, Liaoning & Oct., 2004 & B. bufo gargarizans \\
5 & Shijiazhuang, Hebei & May, 2004 & B. bufo gargarizans \\
6 & Jinan, Shandong & May, 2004 & B. bufo gargarizans \\
7 & Weifang, Shandong & May, 2004 & B. bufo gargarizans \\
8 & Qingdao, Shandong & May, 2004 & B. bufo gargarizans \\
9 & Pingdingshan, Henan & May, 2004 & B. bufo gargarizans \\
10 & Zhengzhou, Henan & May, 2004 & B. bufo gargarizans \\
11 & Yiwu, Zhejiang & May, 2004 & B. bufo gargarizans \\
12 & Jinhua, Zhejiang & May, 2004 & B. bufo gargarizans \\
13 & Changsha, Hunan & May, 2004 & B. bufo gargarizans \\
14 & Yingtan, Jiangxi & May, 2004 & B. bufo gargarizans \\
15 & Foshan, Guangdong & May, 2004 & B. melanostictus \\
16 & Haikou, Hainan & May, 2004 & B. melanostictus \\
17 & Yingtan, Jangxi & May, 2004 & B. melanostictus \\
& & &
\end{tabular}

regions of China and then identified as B. bufo gargarizans and B. melanostictus by Professor Zheng Cui (Table 1). The voucher specimens were deposited in the Pharmacognosy Department, Shenyang Pharmaceutical University (Shenyang, China). The toad venom was collected by squeezing the venom directly from the parotid gland onto a glass plate with a thickness of less than $0.5 \mathrm{~cm}$; it was then dried in the shade. Venom came from at least 40 toads for each sample.

Sample Preparation The dry powder $(0.2 \mathrm{~g})$ of toad venom was refluxed with $90 \mathrm{ml}$ methanol for $1 \mathrm{~h}$. After filtrating and washing the residue, the filtrate was made up to $100 \mathrm{ml}$ by the addition of methanol. This solution was then filtered through a $0.45 \mu \mathrm{m}$ membrane filter, and a volume of $20 \mu \mathrm{l}$ was injected into the HPLC column.

In order to efficiently extract the water-soluble alkaloids and steroid bufogenins, we performed ultrasonic and reflux investigations using chloroform, methanol-chloroform $(1: 1)$, methanol and $75 \%$ methanol in $\mathrm{H}_{2} \mathrm{O}$ as the solvent. The sample preparation methods were evaluated by the extraction efficiency of marker compounds serotonin (1), bufotenidine (4), resibufogenin (12) and cinobufagin (13). It was found that reflux was more efficient than ultrasound. Chloroform and methanol-chloroform $(1: 1)$ were unsuitable for serotonin (1) and bufotenidine (4) extraction (maximum yield 57.2\%, $55.2 \%$ ). Although the yield of serotonin (1) extraction using $75 \%$ methanol was $102.7 \%$ of the amount extracted by methanol, the extractions of bufotenidine (4), resibufogenin (12) and cinobufagin (13) were of a lower amount $(95.5 \%, 85.1 \%, 87.6 \%$, respectively). The extraction times were also considered. The results showed that reflux $(60 \mathrm{~min})$ with methanol as a solvent had an extraction recovery of more than $95 \%$ for the 4 compounds tested by adding standards.

\section{Results and Discussion}

Optimization of HPLC Conditions The optimal chromatographic condition was obtained after testing different mobile phase systems with 3 reversed-phase columns $\left(\mathrm{C}_{8}\right.$, $\mathrm{C}_{18}$ and phenyl-hexyl). The indole alkaloids had good resolution on the phenyl-hexyl column due to its analogical phenyl structure, and the bufogenins were also separated well. Furthermore, among various mobile phases examined, $\mathrm{NaH}_{2} \mathrm{PO}_{4}$-citric acid buffer systems at $\mathrm{pH} 4.0$ showed good separation and peak shape for all analytes. Ion pairing reagents, dodecanesulfonate, heptanesulfonate and methanesulfonate, were tested as the modifiers. It was found that heptanesulfonate leads to good profiles with a sufficient resolution for each indole alkaloid. The concentration of the buffer was reduced to the minimal amount for column protection. Acetonitrile and methanol were also tested for their use as organic solvents in the mobile phase. The former proved unsatisfactory for separation for resibufogenin (12) and cinobufagin (13), while the latter achieved satisfactory separations for all analytes. Finally, by carefully investigating the chromatograms at different wavelengths within the scale of 210 $360 \mathrm{~nm}$, it was found that all the bufogenins and indole alkaloids could be detected with relatively high sensitivities when absorption was measured at $296 \mathrm{~nm}$.

Calibration and Validation We have tested the linearity for compounds $\mathbf{1}-\mathbf{1 4}$ in a range of $2-80 \mu \mathrm{g} / \mathrm{ml}$. The results revealed that the calibration curves of all compounds were linear, and the coefficients of correlation were within $0.996-0.999$. The sample solution was successively injected into the HPLC system and analyzed five times. Intra-day precision not exceeding $1.9 \%$ and $2.8 \%$ was obtained for the retention times and peak areas of all peaks, respectively. The 

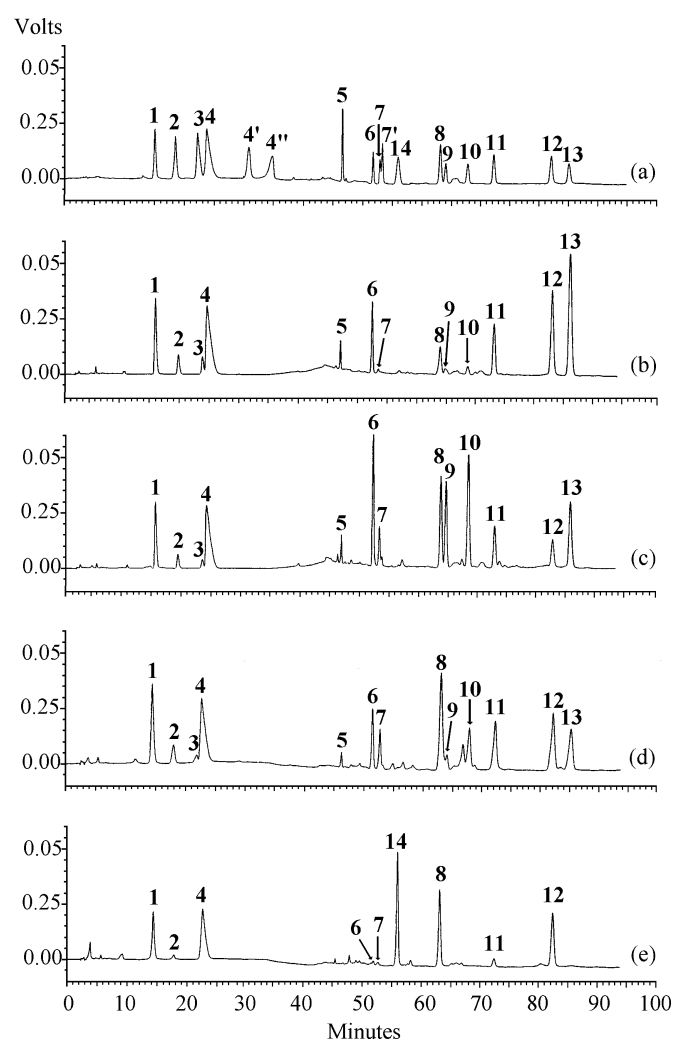

Fig. 2. HPLC Chromatogram of the Different Samples

(a) Compounds isolated from toad venom; (b) toad venom from B. bufo gargarizans (Qiqihaer, north China); (c) toad venom from B. bufo gargarizans (Qingdao, median China); (d) toad venom from $B$. bufo gargarizans (Yingtan, south China); (e) toad venom from B. melanostictus (Yingtan).

inter-day precision of the proposed method, on the basis of analyzing five replicate samples on separate days, was below $2.0 \%$ for retention times and within $3.4 \%$ for peak areas. The stability test was performed with sample solutions for $24 \mathrm{~h}$. The RSD values of retention time and the peak area were less than $2.1 \%$ and $3.5 \%$. Recovery was determined by adding standard compounds to the toad venom samples in appropriate amounts. The analytical recoveries of each compound were between $95 \%$ and $105 \%$.

Identification of Constituents in Chromatograms A standard solution consisting of the 17 isolated compounds with their respective concentrations of $40 \mu \mathrm{g} / \mathrm{ml}$ was analyzed under the developed HPLC conditions. The resulting chromatogram is shown in Fig. 2a. The peaks in the chromatograms of the samples were identified based on their retention times and standard addition. The main peaks in the chromatograms were identified as compounds $\mathbf{1}-\mathbf{1 4}$. Compounds $4^{\prime}, 4^{\prime \prime}$ and $7^{\prime}$ yielded relatively low contents, and were not detected in most of the samples.

Analysis of Toad Venom Samples from Different Origins The newly developed HPLC method was subsequently applied to the quality evaluation of toad venom from different origins. The contents of the compounds were determined from the corresponding calibration curves. Ten batches of commercial toad venom from Shandong, China (the primary production and export region of the drug) were analyzed. The peaks that existed in all chromatograms were assigned as "common peaks". The chromatograms of the commercial toad venom were characterized by 13 "common peaks", which represented compounds $\mathbf{1}-\mathbf{1 3}$. The contents of the 13 major constituents showed small variations. The largest variation was found in resibufogenin (12), having a RSD of $10.54 \%$ for the 10 samples (Table 2). From this study with a limited number of samples, it was found that commercial toad venom samples from the same drug production region exhibited fair consistency in constituents and their contents.

Toad venom samples originating from $B$. bufo gargarizans from different drug production regions of China were investigated using the same procedure. The characteristic 13 "common peaks" could be found in all the chromatograms of each sample. The chromatograph profiles showed high similarities for the alkaloid part, but significant differences for the bufogeins part with respect to their different production regions. The chromatograms (Fig. 2b) of toad venom samples $1-4$, which were collected from north China, showed high contents of resibufogenin $(12,3.65-4.23 \%)$ and cinobufagin $(13,4.44-6.61 \%)$, and extremely low contents of hellebrigenin $(7,0.15-0.29 \%)$, telocinobufagin $(9,0.52-0.75 \%)$ and cinobufotalin $(\mathbf{1 0}, 0.36-0.87 \%)$. Toad venom samples 5-10, which were collected from median China, were characterized by high contents of bufotalin $(8,3.26-4.41 \%)$, telocinobufagin $(\mathbf{9}, 2.23-5.08 \%)$, cinobufotalin $(\mathbf{1 0}, 3.58$ $6.76 \%)$ and cinobufagin $(13,3.03-5.53 \%)$ by their chromatograms, while the content of resibufogenin $(\mathbf{1 2}, 0.27$ $1.35 \%$ ) was lower than in the other samples (Fig. 2c). In the toad venom samples $11-14$ from south China, compounds 10, 12 and 13 were found to have a higher amount of contents, at $1.46-5.45 \%, 1.40-5.97 \%$ and $1.02-2.89 \%$, respectively, and the content of $\mathbf{1 2}$ is higher than $\mathbf{1 3}$, in contract to that from north and median China (Fig. 2d).

The total contents of the bufogenins for toad venom samples from median China $(20.17-26.30 \%)$ were generally higher than those of the other samples (15.12-20.89\%). A different phenomenon was observed for the total alkaloids (4.39-7.20\% vs. 7.81-11.34\%). Bufogenins were the major pharmacologically active constituents in the drug, while $\mathrm{N}$-dimethylated indoleamine derivatives such as bufotenine (3), which have potential hallucinogenic properties, were thought to be a noxious substance and were controlled in many countries. ${ }^{19)}$ Toad venom from Shandong (median China) has been proven to be clinically effective, because it is well tested, having been considered a famous-region drug with an application history of thousands of years in China. This indicated that its chemical constituents were the best for the therapeutic efficacy. In consideration of the historical applications of the drug and the contents of the bioactive ingredients, the quality of toad venom sample from B. bufo gargarizans from median China was recognized as the best, and its chromatograph profiles were proposed as the standardization for quality control of the drug.

The contents of single bufogenins varied widely with respect to the different drug production regions, except for bufalin: the RSD values of its contents exhibited a fair consistency $(19.68 \%)$. This result showed that bufalin was more suitable to be used as a marker compound for the adulterated drug differentiation. Furthermore, the contents of the 14 main compounds in the samples collected from Shenyang in May, August and October were stable, which indicates that the quality of toad venom is not much influenced by the collection period. 
Table 2. Contents (\%) of Main Components of Toad Venom Samples

\begin{tabular}{|c|c|c|c|c|c|c|c|c|c|c|c|c|c|c|c|c|c|}
\hline \multicolumn{2}{|r|}{ Sample } & \multirow{2}{*}{$\begin{array}{r}\text { Comp. } 1 \\
2.26 \pm\end{array}$} & \multirow{2}{*}{$\begin{array}{c}2 \\
0.86 \pm\end{array}$} & \multirow{2}{*}{$\begin{array}{l}3 \\
0.48 \pm\end{array}$} & \multirow{2}{*}{$\begin{array}{l}4 \\
3.32 \pm\end{array}$} & \multirow{2}{*}{$\begin{array}{c}\text { Total } \\
\text { indoles } \\
-6.92 \pm\end{array}$} & \multirow{2}{*}{$\begin{array}{l}5 \\
2.82 \pm\end{array}$} & \multirow{2}{*}{$\begin{array}{l}6 \\
1.75 \pm\end{array}$} & \multirow{2}{*}{$\begin{array}{l}7 \\
1.42 \pm\end{array}$} & \multirow{3}{*}{$\begin{array}{l}\mathbf{8} \\
3.48 \pm \\
0.26\end{array}$} & \multirow{3}{*}{$\begin{array}{l}9 \\
2.54 \pm \\
0.19\end{array}$} & \multirow{3}{*}{$\begin{array}{l}10 \\
6.24 \pm \\
0.44\end{array}$} & \multirow{3}{*}{$\begin{array}{l}11 \\
2.02 \pm \\
0.07\end{array}$} & \multirow{3}{*}{$\begin{array}{l}12 \\
1.06 \pm \\
0.11\end{array}$} & \multirow{3}{*}{$\begin{array}{l}13 \\
4.89 \pm \\
0.41\end{array}$} & \multirow{3}{*}{14} & \multirow{3}{*}{$\begin{array}{c}\begin{array}{c}\text { Total } \\
\text { bufogenins }\end{array} \\
\begin{array}{c}26.23 \pm \\
0.69\end{array}\end{array}$} \\
\hline sp. & coml. & & & & & & & & & & & & & & & & \\
\hline & & 0.20 & 0.09 & 0.03 & 0.20 & 0.35 & 0.17 & 0.13 & 0.13 & & & & & & & & \\
\hline \multirow[t]{18}{*}{$g$} & No. 1 & 2.20 & 0.77 & 0.61 & 4.96 & 8.54 & 0.50 & 0.88 & 0.15 & 1.42 & 0.58 & 0.36 & 2.01 & 3.66 & 6.61 & - & 16.16 \\
\hline & 2 & 2.92 & 0.82 & 0.63 & 4.79 & 9.16 & 0.54 & 1.34 & 0.29 & 1.60 & 0.75 & 0.72 & 1.54 & 3.65 & 4.69 & - & 15.12 \\
\hline & 3 & 2.94 & 0.56 & 0.33 & 5.56 & 9.39 & 0.67 & 1.45 & 0.29 & 1.47 & 0.61 & 0.86 & 1.57 & 4.18 & 4.44 & - & 15.54 \\
\hline & 4 & 3.03 & 0.56 & 0.37 & 5.75 & 9.71 & 0.58 & 1.66 & 0.29 & 1.58 & 0.52 & 0.87 & 1.49 & 4.23 & 4.54 & - & 15.76 \\
\hline & 5 & 2.40 & 0.62 & 0.08 & 1.29 & 4.39 & 1.47 & 1.08 & 1.94 & 4.14 & 2.92 & 3.78 & 1.59 & 1.35 & 4.59 & - & 22.86 \\
\hline & 6 & 2.24 & 0.85 & 0.47 & 3.38 & 6.94 & 2.82 & 1.74 & 1.36 & 3.46 & 2.23 & 6.11 & 1.90 & 0.91 & 5.53 & - & 26.06 \\
\hline & 7 & 3.03 & 0.53 & 0.24 & 1.59 & 5.39 & 0.73 & 0.70 & 0.65 & 3.96 & 3.16 & 3.58 & 1.30 & 1.24 & 4.85 & - & 20.17 \\
\hline & 8 & 1.90 & 0.46 & 0.31 & 4.28 & 6.95 & 0.48 & 3.35 & 2.02 & 3.26 & 5.08 & 5.23 & 1.46 & 1.04 & 3.20 & - & 25.12 \\
\hline & 9 & 2.76 & 0.69 & 0.15 & 3.60 & 7.20 & 1.38 & 1.91 & 2.81 & 3.79 & 3.82 & 6.76 & 1.90 & 0.69 & 3.24 & - & 26.30 \\
\hline & 10 & 2.80 & 0.65 & 0.14 & 3.47 & 7.06 & 0.39 & 3.67 & 1.63 & 3.67 & 4.22 & 6.58 & 1.13 & 0.27 & 3.03 & - & 24.59 \\
\hline & 11 & 5.06 & 0.65 & 0.56 & 5.07 & 11.34 & 0.32 & 0.67 & 1.03 & 1.50 & 3.01 & 5.45 & 1.48 & 4.54 & 2.89 & - & 20.89 \\
\hline & 12 & 3.70 & 0.35 & 0.04 & 3.72 & 7.81 & 0.17 & 0.67 & 0.54 & 1.40 & 1.82 & 1.46 & 1.85 & 5.97 & 2.84 & - & 16.72 \\
\hline & 13 & 4.28 & 0.40 & 0.32 & 4.28 & 9.28 & 0.15 & 1.04 & 7.37 & 1.57 & 1.06 & 3.08 & 1.04 & 1.40 & 1.02 & - & 17.73 \\
\hline & 14 & 2.39 & 0.39 & 0.31 & 4.95 & 8.04 & 0.42 & 2.15 & 2.22 & 2.55 & 1.21 & 3.68 & 1.20 & 2.58 & 1.96 & - & 17.97 \\
\hline & Min & 1.90 & 0.35 & 0.04 & 1.29 & 4.39 & 0.15 & 0.67 & 0.15 & 1.40 & 0.52 & 0.36 & 1.04 & 0.27 & 1.02 & - & 15.12 \\
\hline & Max & 5.06 & 0.85 & 0.63 & 5.75 & 11.34 & 2.82 & 3.67 & 7.37 & 4.14 & 5.08 & 6.76 & 2.01 & 5.97 & 6.61 & - & 26.30 \\
\hline & Average & 2.98 & 0.59 & 0.33 & 4.05 & 7.94 & 0.76 & 1.59 & 1.61 & 2.53 & 2.21 & 3.47 & 1.53 & 2.55 & 3.82 & - & 20.07 \\
\hline & $\mathrm{RSD} \%$ & 28.93 & 26.75 & 58.15 & 33.04 & 22.86 & 93.46 & 59.02 & 115.42 & 44.33 & 68.16 & 66.78 & 19.68 & 70.20 & 38.79 & - & 20.92 \\
\hline \multirow[t]{7}{*}{$m$} & 15 & 1.25 & 0.20 & 0.03 & 2.73 & 4.21 & - & 0.29 & 0.18 & 1.39 & - & - & 0.81 & 0.46 & - & 3.37 & 6.50 \\
\hline & 16 & 1.72 & 0.31 & - & 2.71 & 4.75 & - & 0.46 & 0.45 & 1.01 & - & - & 0.27 & 1.66 & - & 8.47 & 12.32 \\
\hline & 17 & 1.46 & 0.41 & - & 3.28 & 5.16 & - & 0.08 & 0.10 & 2.09 & - & - & 0.19 & 1.38 & - & 7.95 & 11.79 \\
\hline & Min & 1.25 & 0.20 & 0.01 & 2.71 & 4.21 & - & 0.08 & 0.10 & 1.01 & - & - & 0.19 & 0.46 & - & 3.37 & 6.50 \\
\hline & $\operatorname{Max}$ & 1.72 & 0.41 & 0.03 & 3.28 & 5.16 & - & 0.46 & 0.45 & 2.09 & - & - & 0.81 & 1.66 & - & 8.47 & 12.32 \\
\hline & Average & 1.48 & 0.31 & 0.01 & 2.91 & 4.71 & - & 0.28 & 0.24 & 1.50 & - & - & 0.42 & 1.17 & - & 6.60 & 10.20 \\
\hline & RSD\% & 15.94 & 34.25 & 114.56 & 11.13 & 10.12 & - & 68.80 & 75.37 & 36.60 & - & - & 79.66 & 53.81 & - & 42.50 & 31.54 \\
\hline
\end{tabular}

coml., commercial toad vemom (Linyi, Shangdong); g., B. bufo gargarizans; m., B. melanostictus; —, not detected.

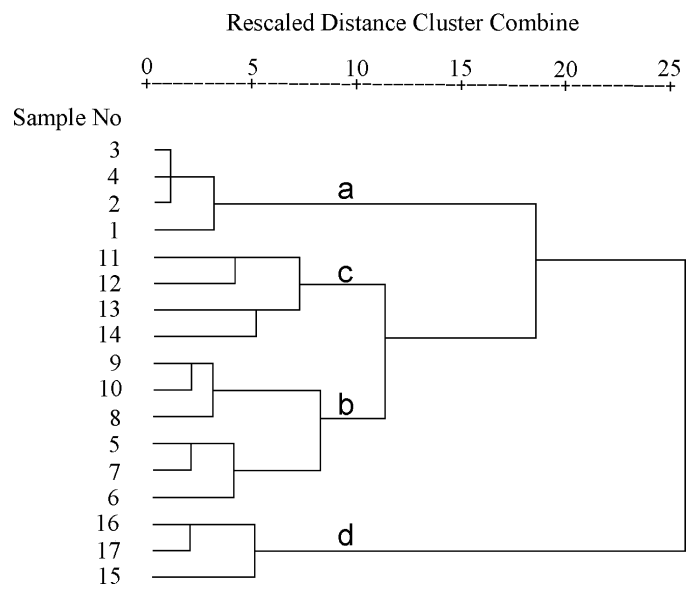

Fig. 3. Dendrogram of Cases Obtained with Hierarchical Clustering Analysis of Toad Venom Samples

Part a corresponds to toad venom from $B$. bufo gargarizans of north China, part $\mathrm{b}$ corresponds to toad venom from $B$. bufo gargarizans of median China, part c corresponds to toad venom from $B$. bufo gargarizans of south China, and part $\mathrm{d}$ corresponds to toad venom from $B$. melanostictus. For details of the samples see Table 1 .

Toad venom samples made from B. melanostictus, another toad species of the drug-providing animals grown only in south China, were analyzed by the developed HPLC method. Their chromatograms were quite different from that obtained from B. bufo gargarizans. There were 9 "common peaks" in the chromatograms, which represented compounds $\mathbf{1}, \mathbf{2}, \mathbf{4}, \mathbf{6}$, 7, 8, 11, 12 and 14. 19-Hydroxy bufalin (14) was the characteristic component, appearing in the highest amounts (3.37$8.47 \%$ ) in the toad venom samples of this species (Fig. 2e). The total content of bufogenins in toad venom from $B$. melanostictus was $6.50-12.32 \%$, which is the half level of that in B. bufo gargarizans. The results indicated that although $B$. melanostictus might be used in the Chinese Pharmacopoeia, its bioactive constituents and the contents of its secretion differed significantly from that of $B$. bufo gargarizans. Therefore, it is imperative that the clinical application and quality control of toad venom from the 2 species of toads should be reconsidered.

Classification of the Toad Venom Samples The contents of 14 main compounds of the toad venom samples from different origins were classified by Hierarchical Cluster analysis (HCA) using the software spss 11.5. To perform cluster analysis we used Ward's method of agglomeration and Euclidean distances to evaluate similarity among the samples. Applying HCA to our data set, we obtained the dendrogram reported in Fig. 3. Four main clusters can be identified: toad venom samples from B. bufo gargarizans of north China, median China and south China, and toad venom samples from B. melanostictus of south China. Samples seem to be clustered on the basis of the toad species and drug production regions.

\section{References}

1) The State Pharmacopoeia Commission of PR China, "Pharmacopoeia of the People's Republic of China (2000 Edn.), Vol. 1," Chemical Industry Publishing House, Beijing, 2000, pp. 316-317.

2) Kopp B., Krenn L., Phytochemistry, 48, 1-29 (1998).

3) Takeda N., Gen. Comp. Endocrinol., 106, 361-373 (1997).

4) Kamano Y., J. Japan. Chem., 24, 339-354 (1970).

5) Hirai Y., Morishita S., Ito C., Sakanashi M., Folia Pharmacologica Japonica, 100, 127-135 (1992).

6) Yoshida S., Kamano Y., Sakai T., Chem. Pharm. Bull., 24, 1714-1717 (1976). 
7) Kamano Y., Yamashita A., Nogawa T., Morita H., Takeya K., Itokawa H., Segawa T., Yukita A., Saito K., Katsuyama M., Pettit G. R., J. Med. Chem., 45, 5440-5447 (2002).

8) Daly J. W., Myers C. W., Science, 156, 970—973 (1967).

9) Kamano Y., Morita H., Takano R., Kotake A., Nogawa T., Hashima H., Takeya K., Itokawa H., Pettit G. R., Heterocycle, 50, 499-503 (1999).

10) Kamano Y., Kotake A., Nogawa T., Tozawa M., Pettit G. R., J. Planar Chromatogr. Modern TLC, 12, 120-123 (1999).

11) Kawahara K., Mikage M., Yakugaku Zasshi, 122, 117-119 (2002).

12) Kubo K., Takakuwa T., Handa K., Yakugaku Zasshi, 97, 274-281 (1977).

13) Yokota Y., Eziri C., Saitou H., Suzuki H., Toyama-ken Yakuji Kenkyusho Nenpo, 17, 83-93 (1989).

14) McClean S., Robinson R. C., Shaw C., Smyth W. F., Rapid Commun.
Mass Spectrom., 16, 346-354 (2002).

15) Ueno A., Ikeya Y., Fukushima S., Noro T., Morinaga K., Kuwano H., Chem. Pharm. Bull., 26, 2411-2416 (1978).

16) Tang Y. Q., Hua J. C., Tian S. H., Wu S. X., Han J. X., Hu G. Y., Ji X. Q., Zou G. W., Guan F., Zhao E. M., Chinese Pharmaceutical J., 25, 138 - 139 (1990).

17) Verpoorte R., Phan Q. K., Baerheim S. A., J. Nat. Prod., 43, 347-352 (1980).

18) Wang J. D., Narui T., Takatsuki S., Hashimoto T., Kobayashi F., Ekimoto H., Abuki H., Niijima K., Okuyama T., Chem. Pharm. Bull., 39, 2135-2137 (1991).

19) Forsstrom T., Tuominen J., Karkkainen J., Scand. J. Clin. Lab. Invest., 61, 547-556 (2001). 